\title{
A graph-theoretical approach for comparison of observational galaxy distributions with cosmological $\mathbf{N}$-body simulations
}

\author{
H. Ueda ${ }^{1,2}$, T. T. Takeuchi ${ }^{2}$, and M. Itoh ${ }^{3}$ \\ ${ }^{1}$ Faculty of Education and Human Studies, Akita University, 1-1 Tegata-gakuen, Akita-shi, Akita 010-8502, Japan \\ 2 National Astronomical Observatory of Japan, 2-21-1 Osawa, Mitaka-shi Tokyo 181-8588, Japan \\ e-mail: takeuchi@optik.mtk.nao.ac.jp \\ 3 Faculty of Economics and Information, Gifu Shotoku Gakuen University, 1-38 Nakauzura, Gifu-shi, Gifu, 500-8288, Japan \\ e-mail:mitoh@gifu.shotoku.ac.jp
}

Received 8 January 2002 / Accepted 4 November 2002

\begin{abstract}
Using a graph-theoretical approach, we compared the galaxy distributions in a flux-limited galaxy sample extracted from the Lyon-Meudon Extragalactic Database ("the LEDA subsample") with those in cosmological $N$-body simulations. To derive information on the density parameter of our Universe, we used CDM simulations with $\left(\Omega_{0}, \lambda_{0}\right)=(0.1,0.9),(0.5$, $0.5),(1.0,0.0)$, and prepared artificial samples. Constellation graphs were constructed from the galaxy distributions in the LEDA subsample and those in these artificial samples, and graph theory was applied. For statistical comparison, the mean absolute deviations of the distribution functions of the eigenvalues of the adjacency matrices were calculated. From our analysis we found that a low-density parameter is preferable, although the LEDA subsample we used in this study is not deep enough to provide a definite estimate of the cosmological parameter set of the Universe.
\end{abstract}

Key words. cosmology: large-scale structure of Universe - methods: numerical, statistic

\section{Introduction}

It is well known that the visual structures of nearby galaxies are filamentary or bubble-like. These structures yield important information about our Universe. Some observations have allowed estimation of the distance of nearby galaxies. The Coma/A1367 survey (Gregory \& Tompson 1978) and the Hercules/A2199 survey (Chincarini et al. 1981) are known as early redshift surveys. Kirshner et al. $(1981,1987)$ discovered the Bootes void which has a diameter of about $60 h^{-1} \mathrm{Mpc}$. These surveys caught the attention of astronomers, and many people have endeavored to produce three-dimensional galaxy maps (for a review, see Rood 1988). The CfA redshift surveys (de Lapparent et al. 1986; Huchra et al. 1990; Geller \& Huchra 1989) which covered wide regions of the sky, revealed the common structure of our universe. SSRS and SSRS2 (da Costa et al. 1988, 1994) which covered galaxies in the southern hemisphere were also important surveys.

In order to derive information about our Universe, it is necessary to quantify galaxy distributions. For this purpose, we here use a graph-theoretical approach (Barrow et al. 1985; Bhavsar \& Ling 1988; Graham et al. 1995; Pearson \& Coles 1995; Krzewina \& Saslow 1996; Gurzadyan \& Kocharyan 1994). Of course, many investigations seeking a powerful

Send offprint requests to: $\mathrm{H}$. Ueda,

e-mail: ueda@ipc.akita-u.ac.jp means to suitably quantify galaxy distributions have been performed (for a review, see Strauss \& Willick 1995). For example, a two-point correlation function is one well known means (Totsuji \& Kihara 1969; Peebles 1980; Davis \& Peebles 1983). From our previous analyses, however, it is evident that a graphtheoretical approach is a more useful method for this purpose as we will see below. The graph-theoretical approach is useful to discriminate differences in galaxy distributions among power-law models (Ueda \& Itoh 1997) and Cold Dark Matter (CDM) models (Ueda \& Itoh 1999). Moreover, this approach is also useful when we apply this to two-dimensional galaxy distributions (Ueda et al. 2001). On the other hand, a two-point correlation function fails to discriminate small differences in galaxy distributions in CDM models. To recognize these differences, it is necessary to calculate higher-order correlation functions (Peebles 1980; Suto \& Mastubara 1994). However, higher-order correlation functions are very difficult to calculate from a real galaxy survey. The graph-theoretical approach is therefore considered to be a suitable approach as a substitute for correlation statistics.

One of the advantages of the graph-theoretical approach is that this attaches importance to nearby pairs. If one thinks over rather distant pairs, the signal/noise ratio becomes very problematic. The necessity of taking into account distant pairs means that correlation function analysis is disadvantageous. The graph-theoretical approach, on the other hand, does not 


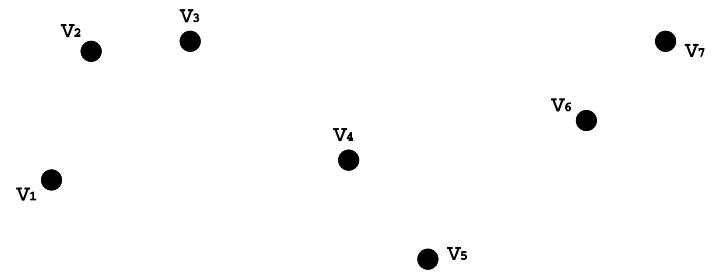

Fig. 1. Example of galaxy distributions.

suffer in the above situations, although this approach lacks clearcut physical interpretations.

In this study, we used a graph-theoretical approach and analyzed an observed galaxy distribution for obtaining information about our real Universe. Though several large redshift surveys have been in progress recently, no dataset from a single survey available at present involves a sufficient volume for our analyses. Thus we prepared a fairly large magnitude limited subsample from the Lyon-Meudon Extragalactic Database (LEDA: Paturel et al. 1997) in this study. Hereafter, we refer to this subsample as "the LEDA subsample".

As simulations intended to derive the density parameters most plausible to describe our Universe, we used cosmological $N$-body simulations with CDM spectra. We analyzed CDM simulations with $\left(\Omega_{0}, \lambda_{0}\right)=(0.1,0.9),(0.5,0.5),(1.0$, $0.0)$, and constructed artificial samples from these simulations. Using a graph-theoretical approach, we compared the galaxy distributions in the LEDA subsample with those in artificial samples.

The remainder of this paper is organized as follows: an overview of the graph-theoretical approach applied here is given in Sect. 2. Observation and simulation data are described in Sect. 3, and in Sect. 4, the results of our analyses are presented. Finally, we summarize our results in Sect. 5.

\section{Overview of a graph-theoretical approach}

In this section, we briefly summarize the graph-theoretical approach applied here. There exist three steps for our analysis: constructing the constellation graphs, calculation of the eigenvalues, and estimating the mean absolute deviation of the eigenvalues.

\section{Step 1}

We first explain how to construct the constellation graphs. To explain a constellation graph, we use an example of the galaxy distributions in Fig. 1. There exits seven galaxies in this figure, and these galaxies are labeled as $v_{1}, v_{2}, \cdots v_{7}$. Now let's connect the nearest neighboring pair of galaxies in this figure. Galaxy $v_{2}$ is the nearest neighbor of galaxy $v_{1}$, so we connect these galaxies at the edges. Galaxy $v_{3}\left(v_{2}\right)$ is the nearest neighbor of galaxy $v_{2}\left(v_{3}\right)$, and galaxy $v_{5}\left(v_{4}\right)$ is the nearest neighbor of galaxy $v_{4}\left(v_{5}\right)$. We also connect these pairs at the edges. Following this procedure, we finally obtain three constellations (see Fig. 2). If we consider the galaxies as vertices and the lines that connect the nearest neighbors as edges, we can regard these
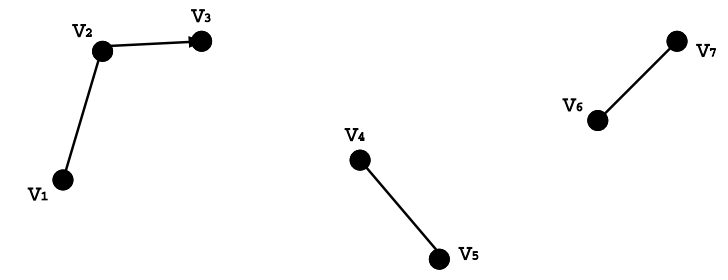

Fig. 2. Results of constellation graphs.

constellations as usual graphs. Anyway, we always construct the constellation graphs from given galaxy distributions.

Before ending our explanation, we mention some features of the constellation graph. As is well known, there exits many types of graphs. The minimal spanning tree (MST) and the Delaunay graph, for example, are famous graphs (Preparata $\&$ Shamos 1985). Why do we choose the constellation graph instead of these famous graphs? In fact, our graph-theoretical approach is based on the pattern recognition of human beings. For example, we recognize the pattern of star distributions as constellations by connecting a clump of stars. Imitating this procedure, we can make galaxy constellations by connecting the nearest neighbors. In order to avoid confusion, we should point out that the constellation graph is different from the MST. This is because only that one graph is constructed according to MST procedure, while there exits some graphs in the constellation graph. (It is known that the constellation graph is a subgraph of the MST.) We also mention the fact that we can uniquely determine the constellation graph. This fact is one of the advantages of our analysis.

\section{Step 2}

We next define the eigenvalues that are derived from the constellation graphs. To carry out this procedure, we introduce the weighted constellation graph. This graph is constructed by assigning the $j$ th power of the edge length $r^{j}$ for each edge. Figure 3 is an example of a weighted constellation graph. This graph is constructed using four vertices $\left(v_{1}, v_{2}, v_{3}, v_{4}\right)$ and three edges. Each edge is weighted the $j$ th power of the edge length $\left(r_{13}^{j}, r_{23}^{j}, r_{34}^{j}\right)$, where $r_{\mathrm{pq}}$ is an edge length between vertices $v_{\mathrm{p}}$ and $v_{\mathrm{q}}$. An artificial parameter $j$ is introduced for changing the rate of weight. We here alter this parameter as $j=0,0.25,0.5, \cdots, 1.75,2.0$. Notice that the $r^{0}$-weighted graph is equivalent to the non-weighted graph, because all of the edge weight is equivalent to one.

Now, we explain the eigenvalues. According to graph theory, an adjacency matrix is assigned to each graph (see Harary 1969; Foulds 1992). We determine an adjacency matrix $A=\left(a_{\mathrm{pq}}\right)$ as

$a_{\mathrm{pq}}= \begin{cases}r_{\mathrm{pq}}^{j} & \text { if vertex } v_{\mathrm{p}} \text { is adjacent to } v_{\mathrm{q}} \\ 0 & \text { otherwise }\end{cases}$ 


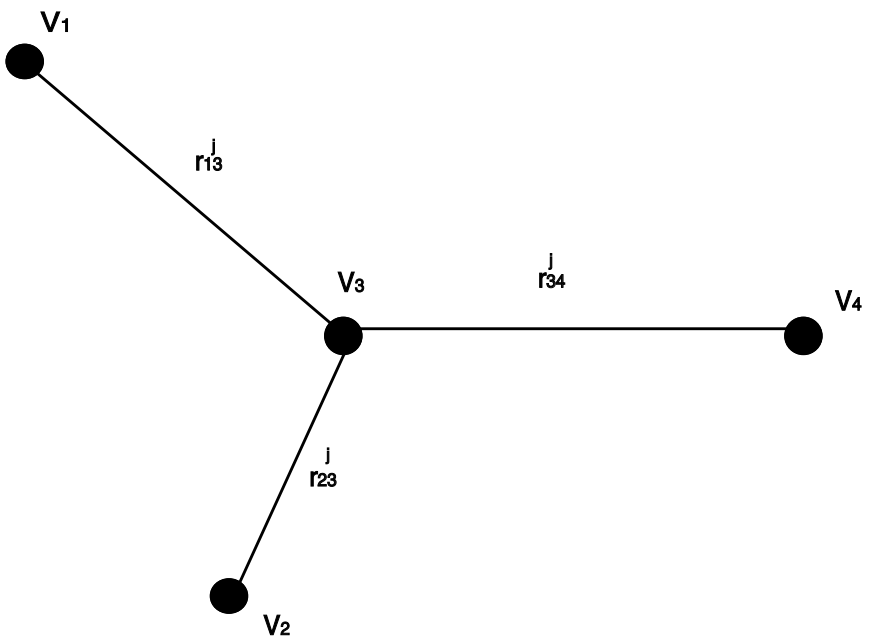

Fig. 3. Example of a graph.

To put it concretely, the adjacency matrix of the graph in Fig. 3 is

$A=\left(\begin{array}{cccc}0 & 0 & r_{13}^{j} & 0 \\ 0 & 0 & r_{23}^{j} & 0 \\ r_{13}^{j} & r_{23}^{j} & 0 & r_{34}^{j} \\ 0 & 0 & r_{34}^{j} & 0\end{array}\right)$.

The adjacency matrix has eigenvalues $e$, which are real numbers, because this matrix is symmetric. The eigenvalues of the matrix in Eq. (2) are

$e=0,0,-\sqrt{r_{13}^{2 j}+r_{23}^{2 j}+r_{34}^{2 j}}, \sqrt{r_{13}^{2 j}+r_{23}^{2 j}+r_{34}^{2 j}}$.

\section{Step 3}

We finally explain the statistical treatment of our graphtheoretical approach. There exist many numbers of the constellation graphs, and each graph has some eigenvalues. From given galaxy distributions, we therefore obtain the distribution function of the eigenvalue $P_{j}(e)$. Of course, the shape of $P_{j}(e)$ differs as the weight $j$ of the constellation graphs changes (see step 2).

To derive a statistical measure, we estimate the mean absolute deviation of $P_{j}(e)$. The definition of the mean absolute deviation $D(j)$ is

$D(j)=\frac{1}{N_{\mathrm{e}}} \sum_{i=1}^{N_{\mathrm{e}}}\left|e_{i}-\bar{e}\right|$,

where $\bar{e}$ and $N_{\mathrm{e}}$ are the mean value and the total number of eigenvalues, respectively. For example, $D(j)$ in Fig. 3 is (see Eq. (3))

$D(j)=\frac{\sqrt{r_{13}^{2 j}+r_{23}^{2 j}+r_{34}^{2 j}}}{2}$.

The advantage of this statistic is extensively described in e.g. Huber (1981). From these procedures, we obtain the mean absolute deviation as a function of the weight index $j$. We use $D(j)$ as a statistical measure to quantify galaxy distributions in each sample.
Table 1. The number of galaxies contained in each sample.

\begin{tabular}{ll}
\hline \hline Sample Name & Number of Galaxies \\
\hline LEDA & 5564 \\
LCDM & 5478 \\
MCDM & 5628 \\
HCDM & 6043 \\
\hline
\end{tabular}

Fig. 4. Galaxy distributions in the LEDA subsample.

\section{Observation and simulation data}

\subsection{The observational data: LEDA}

We constructed a three-dimensional observed galaxy sample from the Lyon-Meudon Extragalactic Database (LEDA: see Paturel et al. 1997 for complete information), which contains a vast number of galaxy redshifts $\sim 10^{6}$. We extracted a magnitude-limited subsample from among the original LEDA galaxies, to be used in estimating nearby galaxy distributions of our Universe. Paturel et al. (1997) provided information on the magnitude completeness of the LEDA, but in this study we adopted a brighter magnitude limit in order to derive firm information. We also checked for incompleteness in the Milky Way region, and rejected any areas suffering from significant extinction. The final limiting magnitude is $13.5 \mathrm{mag}$, and the region of this sample is $1000 \leq v \leq 14000 \mathrm{~km} \mathrm{~s}^{-1}$, and $|b| \geq 15^{\circ}$. As mentioned above, we refer to this subsample as the LEDA subsample. The spatial distribution of the LEDA subsample is presented in Fig. 4. The final data size of the LEDA subsample is shown in Table 1.

To obtain the selection function that is necessary to arrange the $N$-body simulation datasets in the same way as the observational data, we estimated the luminosity function of the LEDA subsample. We used the maximum-likelihood estimation methods of Efstathiou et al. (1988) for this procedure. We found 
that the LEDA luminosity function is well approximated by the Schechter function (Schechter 1976),

$\phi(L) \mathrm{d} L=\phi_{*}\left(\frac{L}{L_{*}}\right)^{\alpha} \exp \left(-\frac{L}{L_{*}}\right) \mathrm{d}\left(\frac{L}{L_{*}}\right)$.

We obtained the parameter values as follows: $\phi_{*}=6 \times 10^{-3}$, $M_{*}=-21.0$, and $\alpha=-1.1$. The characteristic galaxy luminosity $L_{*}$ relates to the characteristic magnitude $M_{*}$ as

$\frac{L_{\odot}}{L_{*}}=10^{-0.4\left(M_{\odot}-M_{*}\right)}$.

Thus we used the above analytical form as the LEDA luminosity function in the following analyses.

\subsection{N-body simulations}

In order to derive information about our Universe, we have used cosmological $N$-body simulations (Ueda \& Itoh 1999; Suginohara et al. 1991). The simulations we considered are designed to probe a density parameter of the CDM cosmological models. Three CDM models with $\left(\Omega_{0}, \lambda_{0}\right)=(0.1,0.9)$, $(0.5,0.5),(1.0,0.0)$ are used, where $\Omega_{0}$ is a density parameter and $\lambda_{0} \equiv \Lambda_{0} /\left(3 H_{0}^{2}\right)$ is a dimensionless cosmological constant. ( $H_{0}$ is the Hubble constant.) Hereafter we call these three models LCDM (Low-density CDM), MCDM (Middle-density CDM), and HCDM (High-density CDM), respectively. These simulations employed $N=262144$ particles, and they were carried out in a cubic volume of $L_{\mathrm{b}}^{3}$ with a periodic boundary condition. All were set with $L_{\mathrm{b}}=100 \mathrm{Mpc}, h=0.7$ where $h$ denotes the Hubble constant $H_{0}$ in units of $100 \mathrm{~km} \mathrm{~s}^{-1} \mathrm{Mpc}^{-1}$. These simulations use a setting of $\sigma_{8}=1.0$ (the standard deviation of the mass fluctuations within an $8 h^{-1} \mathrm{Mpc}$ sphere), and the gravitational softening length is $L_{\mathrm{b}} / 1280$. The masses of an individual particle are $0.52 \times 10^{11} M_{\odot}(\mathrm{LCDM}), 2.6 \times 10^{11} M_{\odot}$ (MCDM) and $5.2 \times 10^{11} M_{\odot}(\mathrm{HCDM})$, roughly equal to a typical galactic mass. In our simple $N$-body simulations, one particle was used to represent one galaxy. The particle distributions of these simulations are in Fig. 5 (LCDM), Fig. 6 (MCDM), and Fig. 7 (HCDM), respectively.

To construct artificial surveys from these simulations, we use a periodic boundary condition. Putting each simulation side by side, we construct a large volume sample. Based on the peculiar velocity of each galaxy, we transfer galaxy distributions from real space to redshift space. By cutting off excess regions, we obtain a map that corresponds to the LEDA subsample. We assign luminosity randomly to each galaxy in this region under the condition that it corresponds to the luminosity function determined by the LEDA subsample. Finally, we select the galaxies whose apparent magnitudes are up to 13.5. The numbers of galaxies that meet the above conditions are also shown in Table 1.

\section{Analysis}

\subsection{Toy model}

Before comparing the galaxy distributions in the LEDA subsample with CDM samples, we examine properties of our

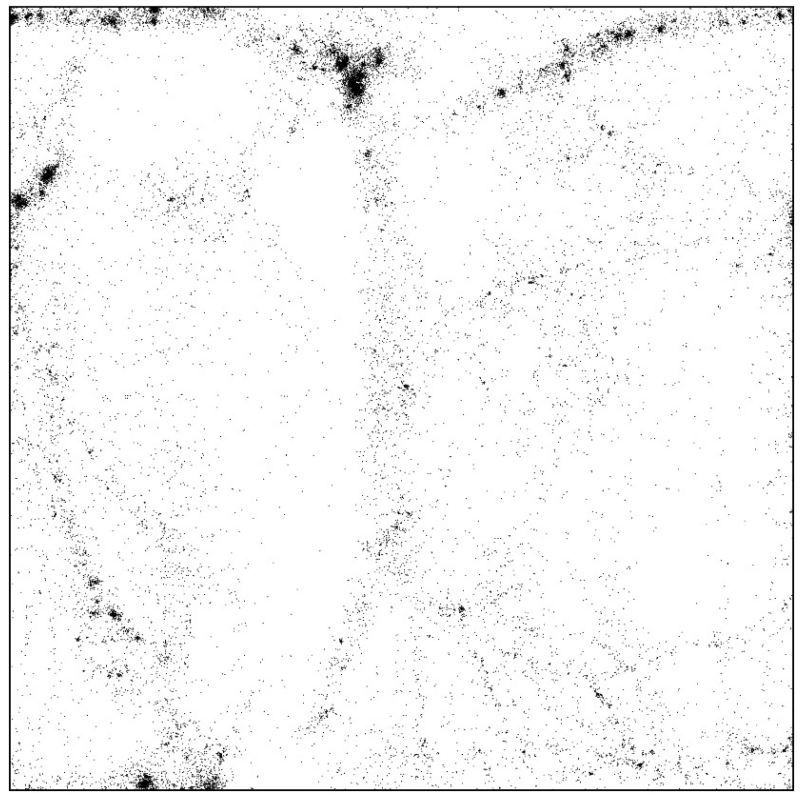

Fig. 5. Particle distributions in the LCDM.

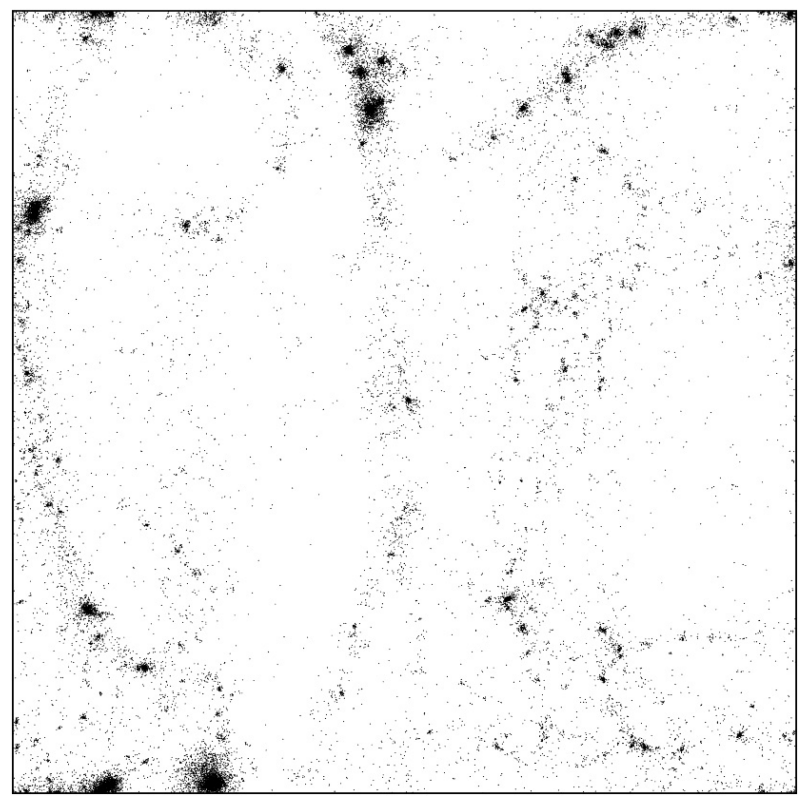

Fig. 6. Particle distributions in the MCDM.

graph-theoretical approach. It is known that this approach is useful to discriminate the difference in galaxy distributions that evolve from different CDM initial spectra (Ueda \& Itoh 1999). As a statistical measure, we have used the mean absolute deviation $D(j)$ of the distribution function of eigenvalues of the adjacency matrices. The mean absolute deviation is not disturbed by the tail of the distribution function. It is therefore considered to be a robust statistical measure.

However, our graph-theoretical approach is difficult to understand in an intuitive way. Of course, we can understand the behavior of this measure to some extent. The mean absolute deviation becomes smaller as the average separations of the galaxy decrease. We can understand this fact from Eq. (5) in Fig. 3 when the index $j$ is positive. To confirm this, we prepare 


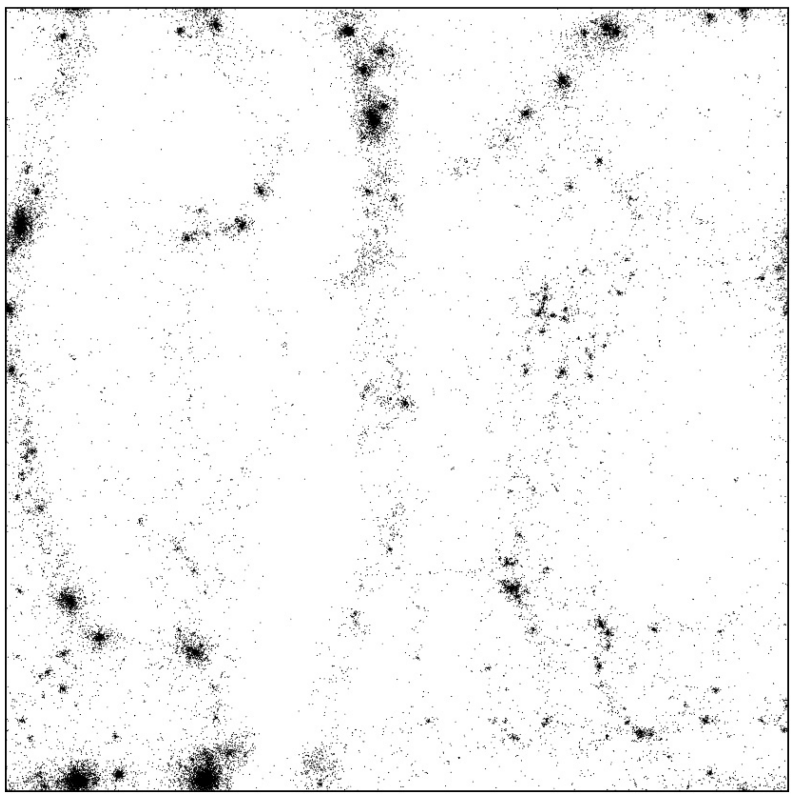

Fig. 7. Particle distributions in the HCDM.

three randomly-distributed galaxy maps. All these samples are constructed by setting ten thousand galaxies in a cube randomly. In the big cube (BCUBE) case, the volume of a cube is $L_{\mathrm{b}}^{3}$. On the other hand, the volume of a cube is $\left(0.9 L_{\mathrm{b}}\right)^{3}$ in the middle cube (MCUBE) and $\left(0.8 L_{\mathrm{b}}\right)^{3}$ in small cube (SCUBE) cases. Constructing the constellation graphs and calculating the eigenvalues, we can estimate the mean absolute deviation $D(j)$ of each sample. Figure 8 is the result of the relative mean absolute deviation $D / D_{\mathrm{BCUBE}}$ of these samples as a function of in$\operatorname{dex} j$. Filled circles, squares, and triangles represent the relative mean absolute deviation of the SCUBE, the MCUBE, and the BCUBE, respectively. The mean separation of galaxies in the SCUBE is apparently the smallest. Then the value of $D_{\text {SCUBE }}$ is also the smallest. From Fig. 8, we obtain clear confirmation of this fact. In previous papers (Ueda \& Itoh 1999), we expressed this fact in other words; the mean absolute deviation becomes smaller as the clustering increases. In any case, we confirm that the galaxy mean separation affects the mean absolute deviation. However, we have to confess that we have had no clear viewpoint of the mean absolute deviation yet.

To derive some insight into our graph-theoretical approach, we apply this to a set of "toy models"; i.e. filamentary patterns, wall-like patterns, and cluster patterns. The filamentary patterns are constructed by arranging galaxies on lines randomly. In this procedure, we set ten lines into a $L_{\mathrm{b}}^{3}$ cube. Using pseudorandom numbers, we put ten thousand galaxies on these lines. In the wall-like patterns, we situate three sheets in a cube, and also put galaxies randomly on these sheets. In this procedure, we also use ten thousand galaxies. The cluster patterns are constructed by preparing one spheroid whose center coincides with the center of a cube, and putting ten thousand galaxies into this spheroid randomly. To treat these models fairly, we set average galaxy separations of these toy models as the same length.

Figure 9 is the relative mean absolute deviation $D / D_{\text {cluster }}$ of toy models as a function of index $j$. Filled circles, squares, and triangles represent the relative mean absolute deviation of

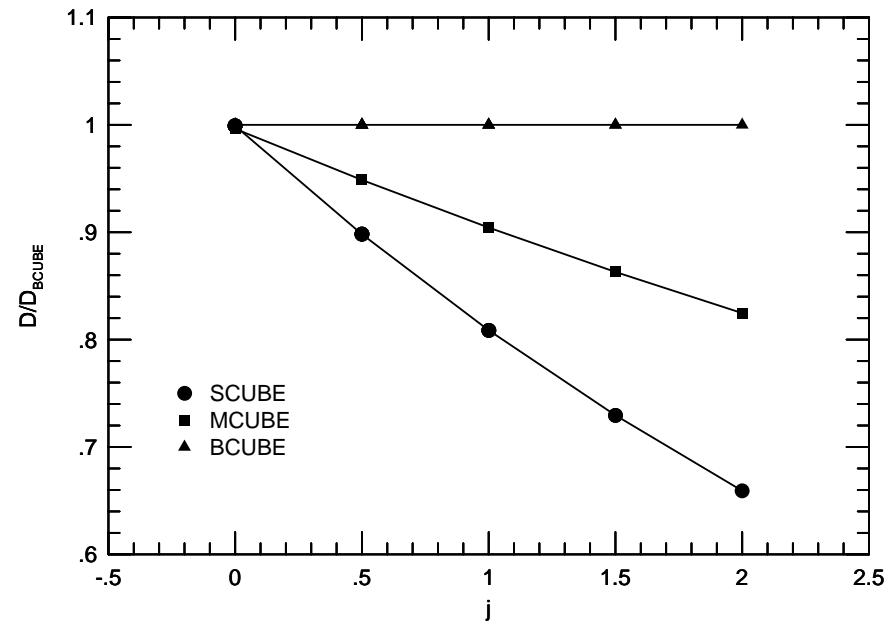

Fig. 8. Relative mean absolute deviation of the distribution functions of the eigenvalues for randomly distributed galaxies.

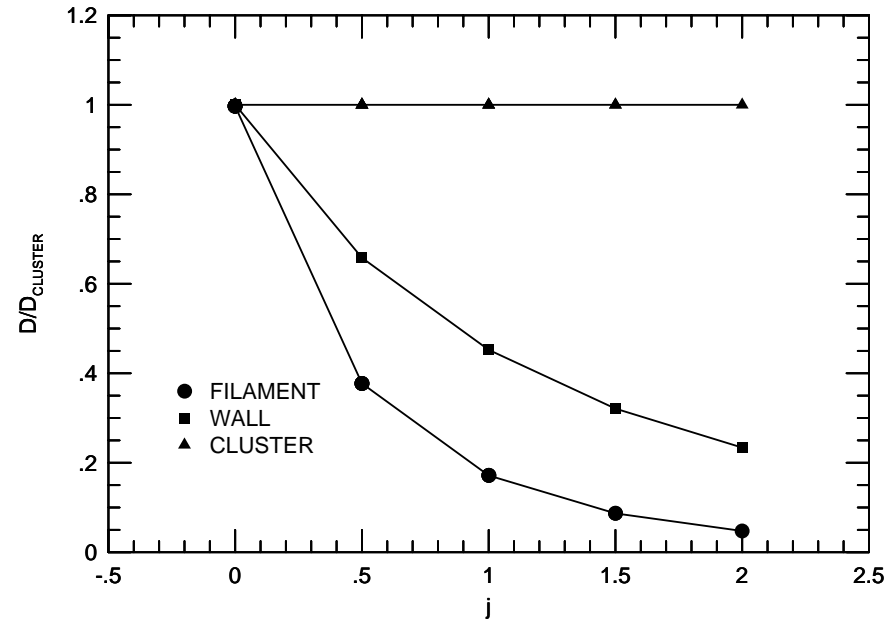

Fig. 9. Relative mean absolute deviation of the distribution functions of the eigenvalues for toy models (filament, wall, cluster type).

filamentary patterns, wall-like patterns, and cluster patterns, respectively. From this analysis, we find that the mean absolute deviation relates to the dimensions of the toy models. $D(j)$ of the filamentary patterns model is the smallest in the range $j>0$. This fact suggests the nature of the constellation graphs. In the filamentary patterns, a galaxy has to be connected either side of the galaxy on a line. In the cluster patterns, on the other hand, a galaxy can be connected to another one in any direction. For this reason, galaxies in the filamentary-like patterns result in small constellation graphs with rather short edges, and galaxies in the cluster patterns result in large constellation graphs with rather long edges. As stated, $D(j)$ becomes smaller as the edge lengths of graphs decrease, so $D(j)$ of filamentary-like patterns is smaller than that in cluster patterns. From our analysis, we find that $D(j)$ reflects the pattern of galaxy distributions in some degree. 


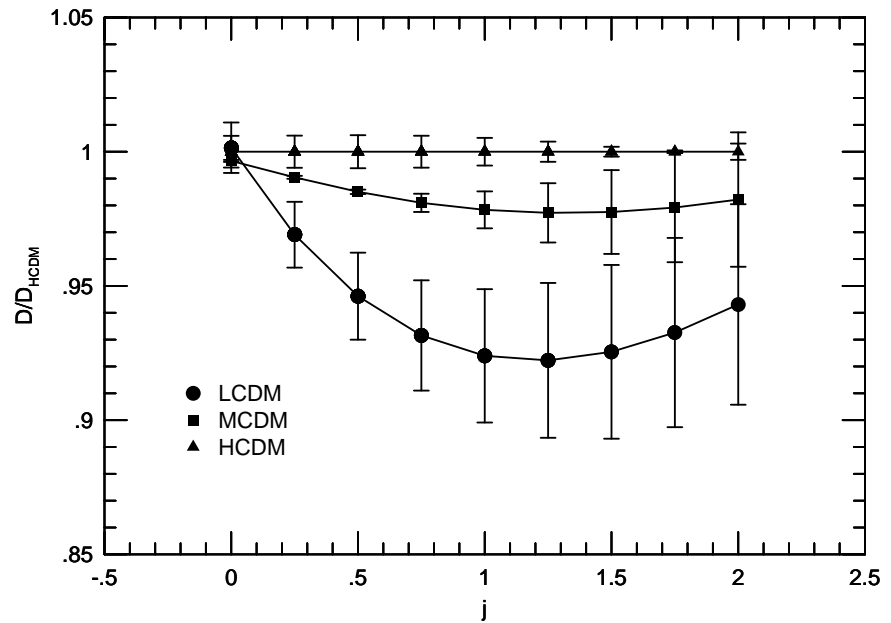

Fig. 10. Relative mean absolute deviation of the distribution functions of the eigenvalues for CDM artificial samples.

\subsection{CDM models}

From previous analyses, our graph-theoretical approach is useful to discriminate the difference in galaxy distributions that evolve from different CDM initial spectra (Ueda \& Itoh 1999). However, one should keep in mind that galaxy distributions of a "survey" selection are very different from the full unbiased particle distributions. It is therefore necessary to check the effectiveness of our graph-theoretical approach when we apply this to CDM artificial samples (see Table 1).

Figure 10 is the relative mean absolute deviation $D / D_{\mathrm{HCDM}}$ of CDM artificial samples as a function of index $j$. Filled circles, squares, and triangles represent the relative mean absolute deviation of LCDM, MCDM, and HCDM, respectively. It should be noted that each artificial sample is separated into a northern and southern hemisphere (see Fig. 4). The error is calculated by treating these two regions differently, i.e. we estimate $D(j)$ of the northern hemisphere and that of the southern hemisphere independently. Following the galaxy numbers, we weight $D(j)$ of these regions and calculate the average and the the standard deviation. This standard deviation is shown as an error bar. In general, the size of the error bars relates to the ratio of the rare graphs in northern and southern hemispheres. A graph that contains rare long edges causes large eigenvalues, and these influence the value of $D(j)$. So the different ratio of the rare graphs dominates the size of the error bars.

From this analysis, we find that the error bars of $D(j)$ grow as $j$ increases. Figure 10 shows that we cannot derive useful information about the difference in the density parameter of these artificial surveys in the region where $j>1.5$. However, the error bars are sufficiently small in the region where $j \leq 1.25$. We therefore conclude that $D(j)$ where $j \leq 1.25$ is useful to quantify these artificial samples. From our analysis, we have confirmed the effectiveness of this graph-theoretical approach; this approach is successful in distinguishing galaxy distributions in three CDM artificial samples. Our analysis shows that the graph-theoretical approach is useful when applied to a three-dimensional map that contains a rather small number of galaxies.

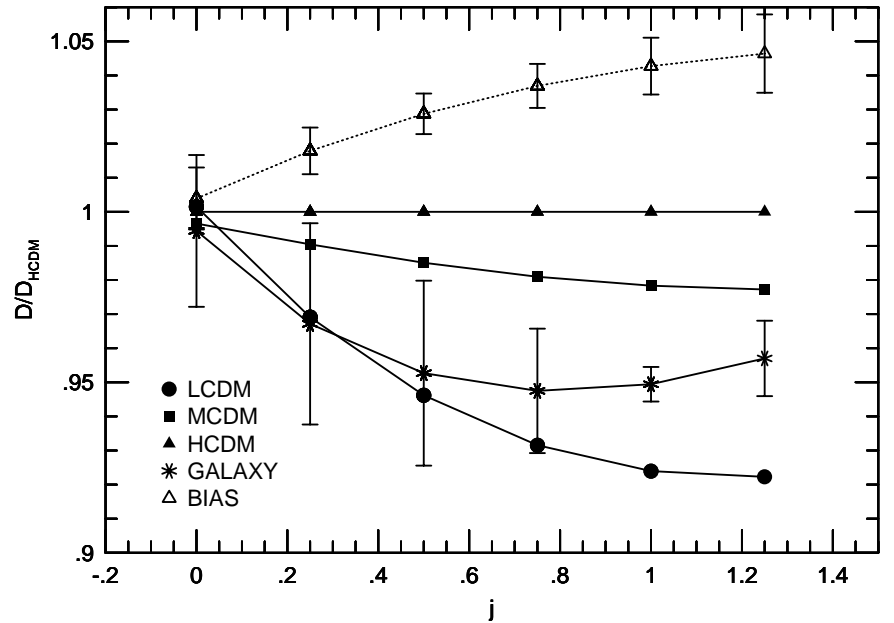

Fig. 11. Relative mean absolute deviation of the distribution functions of the eigenvalues for the LEDA subsample and CDM artificial samples.

\subsection{Observational data and CDM artificial samples}

We now compare the galaxy distributions in the LEDA subsample with those in CDM artificial samples. Figure 11 is the result of the relative mean absolute deviations $D / D_{\mathrm{HCDM}}$ as a function of the index $j(\leq 1.25)$. Filled circles, squares, triangles, and stars represent the relative mean absolute deviation of LCDM, MCDM, HCDM, and the LEDA subsample, respectively. From this figure, we find that $D(j)$ of the LEDA subsample is larger than that of LCDM, and smaller than that of MCDM. Notice that the mean absolute deviation shrinks as the degree of clustering increases. In real space, the degree of galaxy clustering in CDM simulations increases as the density parameter grows. In redshift space, on the other hand, the degree of galaxy clustering decreases as the density parameter increases. This is the result of the finger of God, because the average peculiar velocity of the galaxies in HCDM is larger than that of the galaxies in LCDM.

According to the above discussion, $D(j)$ in redshift space shrinks as the density parameter decreases. It is therefore concluded that the LEDA subsample agrees with a CDM model of low-density type. In particular, in our analyses, the density parameter of our Universe is predicted to be $0.1 \leq \Omega_{0} \leq 0.5$.

Before ending this subsection, it is necessary to consider a galaxy-biasing effect. Until now, we have avoided this effect and assumed $\sigma_{8} \equiv 1 / b$ as being equal to one ( $b$ is a bias parameter). Under this condition, we derive a constraint of the density parameter as less than one. Is this constraint still effective if we set $\sigma_{8} \neq 1$ ? To see this, we also perform a cosmological $N$-body simulation with $\left(\Omega_{0}, \lambda_{0}, \sigma_{8}\right)=(1.0,0.0,0.52)$, and make an artificial sample (Eke et al. 1996). The number of galaxies in this artificial sample is 6087 . We calculate the mean absolute deviations, the result of which is also shown in Fig. 11 (open triangles). In general, strength of clustering in $\sigma_{8}<1$ type simulation (we call this model "BIAS") is weak, and remember that the mean absolute deviation becomes larger as the clustering decreases. One soon notices that the value of $D(j)$ in BIAS is larger than that in HCDM. In fact, we can confirm this 
trend in Fig. 11. It is therefore believed that the CDM model with $\sigma_{8}<1$ type has no possibility of decreasing a disagreement between the standard CDM model and observation.

\section{Summary}

In this paper, we have used a graph-theoretical approach for comparing galaxy distributions in an LEDA subsample with those in $N$-body simulations. In order to derive information on the density parameter of our Universe, we examined CDM simulations with $\left(\Omega_{0}, \lambda_{0}\right)=(0.1,0.9),(0.5,0.5),(1.0,0.0)$. Our analysis strongly suggests that the density parameter of our Universe is less than one. More precisely, on the basis of our analyses, the density parameter is predicted to be $0.1 \leq \Omega_{0} \leq$ 0.5 . This result is consistent with recent research findings. We also found that the standard CDM model with $\sigma_{8}<1$ type also disagrees with observation.

We do not discuss more stringent restrictions on the density parameter of our Universe, or the validity of the CDM model. This is because the LEDA subsample is considered to be insufficient for a detailed statistical treatment. To see this, we estimate $D(j)$ from galaxy distributions in the northern and southern hemispheres of the LEDA subsample. Unfortunately, the mean absolute deviations for the northern and southern hemispheres are rather different from each other, which suggests that the data is not deep enough to ignore the inhomogeneity of galaxy distributions in the Local Universe. If we want to carry out detailed analyses, we have to obtain a more complete sample. Efforts to determine the three-dimensional position of nearby galaxies will be completed in the near future. For example, the Sloan Digital Sky Survey, and the 2 Degree Field observations are famous projects. However, it takes some time to obtain these next generation surveys.

Although complete three-dimensional galaxy maps that suit statistical treatments were lacking, we were able to derive the restrictions on the density parameter of our Universe. In the course of our analysis, we again came to appreciate the usefulness of the graph-theoretical approach. This is one of the most powerful and appropriate methods available to quantify galaxy distributions. We therefore look forward to having the opportunity to apply this method to the next generation surveys.

Acknowledgements. We would like to thank the Yukawa Institute for allowing us to use their computer system. We are also grateful to the LEDA team at the CRAL-Observatoire de Lyon (France), who allowed us to access the Lyon-Meudon Extragalactic Database (LEDA).
The present analyses were carried out on SUN SPARC stations at the Faculty of Education and Human Studies, Akita University and Yukawa Institute for Theoretical Physics, Kyoto University.

\section{References}

Barrow, J. D., Bhavsar, S. P., \& Sonoda, D. H. 1985, MNRAS, 216, 17

Bhavsar, S. P., \& Ling, N. E. 1988, ApJ, 331, 63

Chincarini, G., Rood, H. J. \& Thompson, L. A. 1981, ApJ, 249, 47

da Costa, L. N., et al. 1988, ApJ, 327, 544

da Costa, L. N., et al. 1994, ApJ, 424, 1

Davis, M., \& Peebles, P. J. E. 1983, ApJ, 267, 465

de Lapparent, V., Geller, M. J., \& Huchra, J. P. 1986, ApJ, 324, 1

Eke, V. R., Cole, S., \& Frenk, C. S. 1996, MNRAS, 282, 263

Efstathiou, G., Ellis, R. S., \& Peterson, B. A. 1988, MNRAS, 232, 431

Foulds, L. R. 1992, Graph Theory Application (Springer-Verlag, New York)

Geller, M. J., \& Huchra, J. P. 1989, Science, 246, 897

Graham, M. J., Clowes, R. G., \& Campusano, L. E. 1995, MNRAS, 275,790

Gregory, S. A., \& Tompson, L. A. 1978, ApJ, 222, 784

Gurzadyan, V. G., \& Kocharyan, A. A. 1994, Paradigms of the LargeScale Universe (Gordon \& Breach)

Harary, F. 1969, Graph Theory (Addison-Wesley Publishing Company, Inc; Massachusetts)

Huber, P. J. 1981, Robust Statistics (John Wiley \& Sons, New York)

Huchra, J. P., Geller, M. J., de Lapparent, V., \& Corwin, H. G. Jr. 1990, ApJS, 72, 433

Kirshner, R. P., Oemler, A. Jr., Schechter, P. L., \& Shectman, S. A. 1981, ApJ, 248,57

Kirshner, R. P., Oemler, A. Jr., Schechter, P. L., \& Shectman, S. A. 1987, ApJ, 314,493

Krzewina, L. G., \& Saslaw, W. C. 1996, MNRAS, 278, 869

Paturel, G., Andernach, H., Bottinelli, L., et al. 1997, A\&AS, 124, 109

Pearson, R. C., \& Coles, P. 1995, MNRAS, 272, 231

Peebles, P. J. E. 1980, The Large Scale Structure of the Universe (Princeton University Press, Princeton)

Rood, H. J. 1988, ARA\&A, 26, 245

Schechter, P. 1976, ApJ, 203, 297

Strauss, M. A., \& Willick, J. A. 1995, Phys. Rep., 261, 271

Suginohara, T., Suto. Y., Bouchet, F. R., \& Hernquist, L. 1991, ApJS, 75,631

Suto, Y., \& Matsubara, T. 1994, ApJ, 420, 504

Totsuji, H., \& Kihara, T. 1969, PASJ, 21, 221

Ueda, H., \& Itoh, M. 1997, PASJ, 49, 131

Ueda, H., \& Itoh, M. 1999, ApJ, 526, 560

Ueda, H., Takeuchi, T. T., \& Itoh, M. 2001, PASJ, 53, 381 